IRA-International Journal of Education \& Multidisciplinary Studies

ISSN 2455-2526; Vol.03, Issue 03 (2016)

Institute of Research Advances

http://research-advances.org/index.php/IJEMS

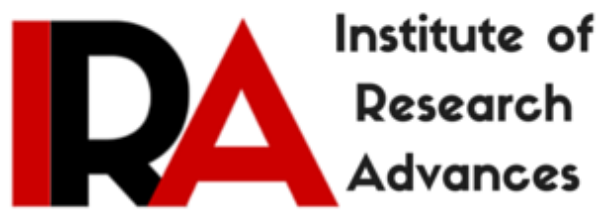

\title{
Estimates of Healthcare - An Investigation with Probit Model
}

\section{Dr. Shivesh}

Asst. Professor, M.B.A. Agri Business,

RGSC, Banaras Hindu University, UP, Varanasi, India.

DOI: http://dx.doi.org/10.21013/jems.v3.n3.p26

\section{How to cite this paper:}

Shivesh. (2016). Estimates of Healthcare - An Investigation with Probit Model. IRA International Journal of Education and Multidisciplinary Studies (ISSN 2455-2526), 3(3). doi:http://dx.doi.org/10.21013/jems.v3.n3.p26

(C) Institute of Research Advances

\section{(c) EY-NC}

This work is licensed under a Creative Commons Attribution-Non Commercial 4.0 International License subject to proper citation to the publication source of the work.

Disclaimer: The scholarly papers as reviewed and published by the Institute of Research Advances (IRA) are the views and opinions of their respective authors and are not the views or opinions of the IRA. The IRA disclaims of any harm or loss caused due to the published content to any party. 


\begin{abstract}
Health systems with universal health care coverage aspire to achieve horizontal equity, commonly defined as equal access to health care services for equal need. While equal access presupposes that individuals are given equal opportunities to access services, the goal of equal utilization for equal need implies a different set of conditions. Although inequity in utilization may not solely reflect inappropriate or unfair differentials in service use, revealing instead different preferences or culture (Oliver and Mossialos 2004), it is the measure of equity most commonly studied to date. In this paper we investigate utilization of health services in the absence of data on access, consistent with other studies. There has been considerable research in the area of equity in utilization of health services in past decades focussing on the general adult population and using cross-sectional data sources. Studies in the India are not conclusive, although they suggest that utilization of primary care and hospital services, after adjusting for health care need, is equitable or pro-poor, whereas preventive and specialist care tends to favour the better off. Some empirical studies and reviews of the literature contend that GP and inpatient service use is pro-poor (O'Donnell and Propper 1991; Propper 1998; Goddard and Smith 2001; Dixon et al 2006. Several European studies have investigated socio-economic differences in the use of services among the older population after adjusting for self-reported health status.
\end{abstract}

\title{
Introduction
}

Health systems with universal health care coverage aspire to achieve horizontal equity, commonly defined as equal access to health care services for equal need. While equal access presupposes that individuals are given equal opportunities to access services, the goal of equal utilization for equal need implies a different set of conditions. Although inequity in utilization may not solely reflect inappropriate or unfair differentials in service use, revealing instead different preferences or culture (Oliver and Mossialos 2004), it is the measure of equity most commonly studied to date. In this paper we investigate utilization of health services in the absence of data on access, consistent with other studies. There has been considerable research in the area of equity in utilization of health services in past decades focussing on the general adult population and using cross-sectional data sources. Studies in the India are not conclusive, although they suggest that utilization of primary care and hospital services, after adjusting for health care need, is equitable or pro-poor, whereas preventive and specialist care tends to favour the better off. Some empirical studies and reviews of the literature contend that GP and inpatient service use is pro-poor (O'Donnell and Propper 1991; Propper 1998; Goddard and Smith 2001; Dixon et al 2006. Several European studies have investigated socio-economic differences in the use of services among the older population after adjusting for self-reported health status.

The purpose of the present study is to contribute to the literature on inequity in older people in The India by measuring income-related inequity after controlling for differences in need in the use of GP, outpatient, inpatient and dental services with longitudinal data from the period 1997 to 2003. The advantages of a panel structure are various. On the one hand, it allows us to consider the dynamic structure of the relationship between health, income and health care use; and on the other, it allows us to control for unobserved cross-section heterogeneity.

\section{Data description}

This study was conducted using data from the Indian Household Panel Survey (IHPS).1 Using panel data allows us to correct for unobserved heterogeneity. The BHPS is a longitudinal cohort survey of adult members of a nationally representative sample of Indian households, including Scotland, Northern Ireland and Wales. The latest wave of the BHPS with available data was collected in 2003 (Wave 13).

The survey collects data from all adult members of the household. Those in the initial sample are followed until they refuse to participate, die, or are lost to follow-up. The present study included all 
individuals aged 65 or over in the period 1997 to 2003, as 1997 was the first year that included information on private medical insurance coverage. Only those with complete responses were included in the analysis, therefore those with proxy respondents (due to inability to respond themselves) were excluded. The percentage of proxy respondents is around $2 \%$.

Socio-economic variables include educational level, housing tenure and income. Educational qualifications are separated into three groups: no qualifications; non-advanced qualifications (including apprenticeships and secondary education); and advanced qualifications (higher degree, first degree, teaching and 'other' qualifications). Housing tenure is included as a categorical variable: whether the individual owns his or her home, rents from a local or housing authority, or rents privately.

Income is measured as gross household income in the last month, which is derived from disaggregated income sources including labour and non-labour income, transfer income, investment income, benefit income and pension income. Income is equivalized for household composition using the BHPS equivalization scale.

Other socio-demographic factors are included in the models. Individual coverage with private medical insurance (PMI; either through an employer/previous employer or an individual plan) is included in the analysis. Region and time dummies are included in order to capture crude differences in health care supply, and also possible changes over timeMeasures of need are examined separately and are approximated from several health indicators, in order to most accurately capture health care need. Information on self-reported health status came from the following question: "Please think back over the last 12 months about how your health has been.

Compared to people of your own age, would you say that your health has on the whole been: excellent, good, fair, poor, or very poor?" In wave 9 of the BHPS (1999), self-assessed health is measured differently with the question: "In general would you say your health is: excellent, very good, fair, or poor". In light of the inconsistency in the measurement of self-assessed health throughout the panel, we created only three categories: (1) excellent or very good; (2) good or fair; (3) poor or very poor (for a detailed discussion of this methodological problem, see HernandezQuevedo et al 2005). Additional health indicators include whether or not the respondent has any of the fifteen listed health problems in the survey4, which is used to create a variable indicating the presence of three or more health problems5In addition to the above indicators of health status, other demographic variables related to need were also considered: age and sex. Age is measured at the time of the interview, and is grouped into 5-year age bands: 65-69; 70-74; 75-79; 80-84, and 85 and over. In the case of dental care, only age and sex were considered for the estimates of "needrelated' utilization. The health variables were not used as proxies for need since a preliminary analysis showed that healthy, younger individuals are more likely to access dental care than those who have more self-reported health problems.

Health service use is measured by the following questions: 'approximately how many times have you talked to, or visited a GP or family doctor about your own health [in the past year]? Have you yourself made use of hospital consultant/outpatient services [in the past year]? Have you been in hospital or clinic as an in-patient overnight or longer [in the past year]? Have you had a dental check-up [in the past year]?

The proportion of people who used any of the above health care services are outlined in Table 1 for each year in addition to the sample sizes and average age of the population under analysis. The increase in sample size over the period results both from individuals ageing thus entering the age $65+$ age group, and additional individuals being included in the sample as they enter a household with an original sample member. 
Table 1. Description of the data

\begin{tabular}{lllllll} 
Year & $\begin{array}{l}\text { Sample } \\
\text { size }\end{array}$ & Mean age & $\begin{array}{l}\text { \% visited } \\
\text { GP }\end{array}$ & $\begin{array}{l}\text { \% visited } \\
\text { outpatient }\end{array}$ & $\begin{array}{l}\text { \% admitted } \\
\text { to hospital }\end{array}$ & $\begin{array}{l}\text { \% visited } \\
\text { dentist }\end{array}$ \\
\hline 1997 & 1,939 & 74.2 & 84.2 & 32.9 & 15.38 & 37.2 \\
1998 & 1,914 & 74.5 & 84.7 & 36.7 & 17.08 & 39.7 \\
1999 & 2,788 & 74.2 & 84.3 & 39.1 & 18.26 & 38.8 \\
2000 & 2,737 & 74.0 & 85.8 & 41.5 & 16.92 & 40.6 \\
2001 & 3,293 & 74.2 & 86.0 & 40.3 & 16.52 & 42.9 \\
2002 & 2,817 & 74.2 & 86.1 & 41.5 & 15.58 & 45.6 \\
2003 & 2,786 & 74.3 & 85.6 & 43.3 & 15.61 & 45.2 \\
\hline
\end{tabular}

\section{Methods}

In order to measure income-related inequality and inequity, concentration (inequality) indices were calculated according to the indirect standardization approach using a 'convenient regression' The inequality index $(\mathrm{Cm})$ would reach zero if all individuals had equal probability of seeking health care, regardless of income; the inequity index (HI) would be zero if after controlling for differences in need across income groups, individuals on different income would have equal probability of service use. The HI would be positive (negative) if higher income individuals were more (less) likely to use health care than those with lower income, after standardising for need. The index of the distribution of need according to income $(\mathrm{Cn})$ is negative if greater need is concentrated among the worse off, and vice versa. In other words, the horizontal inequity index addresses the question: after controlling for differences in need (as measured by health status and other need-related demographic factors) ${ }^{6}$ across income groups, are individuals on higher income more likely to use health care services than lower income comparators?

By using seven waves of the IHPS (unbalanced panel) it is possible to correct for individualspecific unobservable effects in the error term (Wooldridge, 2002). Estimates of each health care use (GP, specialist, hospital or dental care) are obtained by using a Probit Model where the dependent variable $y_{i}$ equals one if the individual used health care or zero otherwise.

$$
\begin{array}{cc}
y=1 & \text { if } y^{*}>0 \\
y=0 & \text { otherwise }
\end{array}
$$

where,

$$
y_{i t} *=X^{\prime}{ }_{i t} \beta+Z^{\prime}{ }_{i t} \delta+a_{i}+\varepsilon_{i t}
$$

$X$ and $Z$ are the vectors of need and non-need variables, and the error term is represented by two components, $\mathrm{a}_{\mathrm{i}}$ and $\varepsilon_{\mathrm{it}}$. The former is the individual effect that is treated as random while the latter is the idiosyncratic disturbance.

The random effect model will provide efficient estimates of _and $\beta \delta$ and will also provide information on how much of the variability in health care use is due to individual effect.

Under the assumptions that $\alpha_{\mathrm{i}}$ and $\varepsilon_{\mathrm{it}}$ are normally distributed and independent of $\mathrm{X}$, it is possible to Integrate $\alpha$ i out to obtain the sample log-likelihood function: 


$$
\ln L=\sum_{i=1}^{n} \ln \quad \int_{-\infty}^{+\infty} \prod^{T}\left(\Phi\left[\left(2 y_{i t-1}\right)\left(X^{\prime}{ }_{i t} \quad \beta+Z^{\prime}{ }_{i t} \quad+a\right)\right] f(a) d a\right)
$$

This integral can be approximated by the Gauss-Hermite quadrature, and if we assume that $\alpha_{i}$ is normally distributed with mean 0 and variance_$\sigma^{2}$, then the contribution of each individual to the sample likelihood function can be written as:

$$
L=\int_{-\infty}^{+\infty}\left(1 / \sqrt{2 \pi \sigma_{a}^{2}}\right) \exp \left(-a^{2} / 2 \sigma_{a}{ }^{2}\right)\left\{\prod_{t=1}^{T} \Phi\left[\left(2 y_{i t-1}\right)\left(X^{\prime}{ }_{i t} \beta+Z^{\prime}{ }_{i t}+a\right)\right]\right\} d a
$$

The random effect probit model estimates are obtained using STATA 9.0.

\section{Results}

The results of the random effects probit models for the probability of each health care service use during the period 1997 to 2003 are reported in Table 2. The health care need indicators are most strongly associated with health service use in all health service areas except dental care, where younger age groups and women are more likely to seek dental care.

Among the socio-economic factors, holding private medical insurance (PMI) is significantly associated with all four health service areas, in particular with dental care where individuals with PMI are 50\% more likely to have seen a dentist in the past year. Home ownership and higher educational qualifications are significantly associated with outpatient and, more strongly, dental services. Regional effects are less significant. Compared to those living in London, individuals in Northern Ireland are less likely to have an outpatient visit. Those living in Scotland have increased probability of a GP visit and an inpatient stay. Finally, individuals in Lucknow ,Varanasi, Mirzapur are less likely than those living in Lucknow to have a dental check-up. 
Table 2. Factors associated with health service use (coefficients and standard errors)

\begin{tabular}{|c|c|c|c|c|c|c|c|c|}
\hline & \multicolumn{2}{|c|}{ GP visit } & \multicolumn{2}{|c|}{ Outpatient } & \multicolumn{2}{|c|}{ Inpatient } & \multicolumn{2}{|c|}{ Dentist } \\
\hline & Coef & SE & Coef & SE & Coef & SE & Coef & SE \\
\hline Log of income & 0.03 & 0.03 & 0.08 & 0.03 & 0.06 & 0.03 & 0.25 & 0.04 \\
\hline \multicolumn{9}{|c|}{ Health/demographic indicators (need) } \\
\hline Fair health & 0.53 & 0.04 & 0.48 & 0.03 & 0.51 & 0.03 & & \\
\hline Poor health & 0.88 & 0.07 & 0.88 & 0.04 & 1.08 & 0.04 & & \\
\hline$>3$ health problems & 0.51 & 0.05 & 0.38 & 0.03 & 0.17 & 0.03 & & \\
\hline Health limits behaviour & 0.20 & 0.06 & 0.13 & 0.04 & 0.18 & 0.04 & & \\
\hline Disabled & 0.06 & 0.04 & 0.13 & 0.03 & 0.14 & 0.03 & & \\
\hline Female & 0.13 & 0.05 & 0.00 & 0.04 & -0.18 & 0.04 & 0.28 & 0.07 \\
\hline $70-74$ & 0.03 & 0.05 & 0.04 & 0.04 & 0.06 & 0.04 & -0.22 & 0.05 \\
\hline $75-79$ & 0.11 & 0.06 & 0.00 & 0.04 & 0.20 & 0.04 & -0.59 & 0.07 \\
\hline $80-84$ & 0.08 & 0.07 & 0.07 & 0.05 & 0.27 & 0.05 & -0.89 & 0.09 \\
\hline $85+$ & 0.04 & 0.08 & 0.01 & 0.06 & 0.33 & 0.06 & -1.27 & 0.12 \\
\hline \multicolumn{9}{|c|}{ Socioeconomic and regional indicators } \\
\hline Not married & -0.23 & 0.07 & -0.09 & 0.05 & 0.07 & 0.05 & -0.04 & 0.10 \\
\hline Widowed & -0.14 & 0.05 & -0.09 & 0.04 & 0.12 & 0.04 & -0.27 & 0.07 \\
\hline Smoker & -0.28 & 0.06 & -0.24 & 0.04 & -0.20 & 0.05 & -0.72 & 0.08 \\
\hline Owns home & 0.06 & 0.05 & 0.13 & 0.04 & -0.02 & 0.04 & 0.66 & 0.08 \\
\hline Rents privately & 0.10 & 0.10 & 0.05 & 0.07 & 0.01 & 0.08 & 0.36 & 0.12 \\
\hline PMI Non- & 0.23 & 0.08 & 0.17 & 0.06 & 0.13 & 0.06 & 0.53 & 0.10 \\
\hline advanced & 0.00 & 0.05 & 0.15 & 0.04 & 0.01 & 0.04 & 0.89 & 0.08 \\
\hline Advanced qualifications & 0.08 & 0.07 & 0.17 & 0.05 & 0.02 & 0.05 & 1.46 & 0.10 \\
\hline Lucknow & 0.17 & 0.12 & 0.05 & 0.09 & -0.03 & 0.09 & 0.28 & 0.19 \\
\hline Chandauli & 0.14 & 0.11 & 0.01 & 0.08 & 0.01 & 0.08 & -0.21 & 0.17 \\
\hline Varanasi & 0.09 & 0.12 & -0.09 & 0.09 & 0.05 & 0.09 & -0.43 & 0.18 \\
\hline Mirzapur & 0.31 & 0.12 & 0.08 & 0.09 & 0.23 & 0.09 & -0.68 & 0.18 \\
\hline Time dummies & 0.29 & 0.12 & -0.32 & 0.09 & 0.13 & 0.09 & -0.30 & 0.18 \\
\hline
\end{tabular}


Figure 1. Horizontal inequity (and 95\% confidence intervals) in health service use among over $65 \mathrm{~s}$

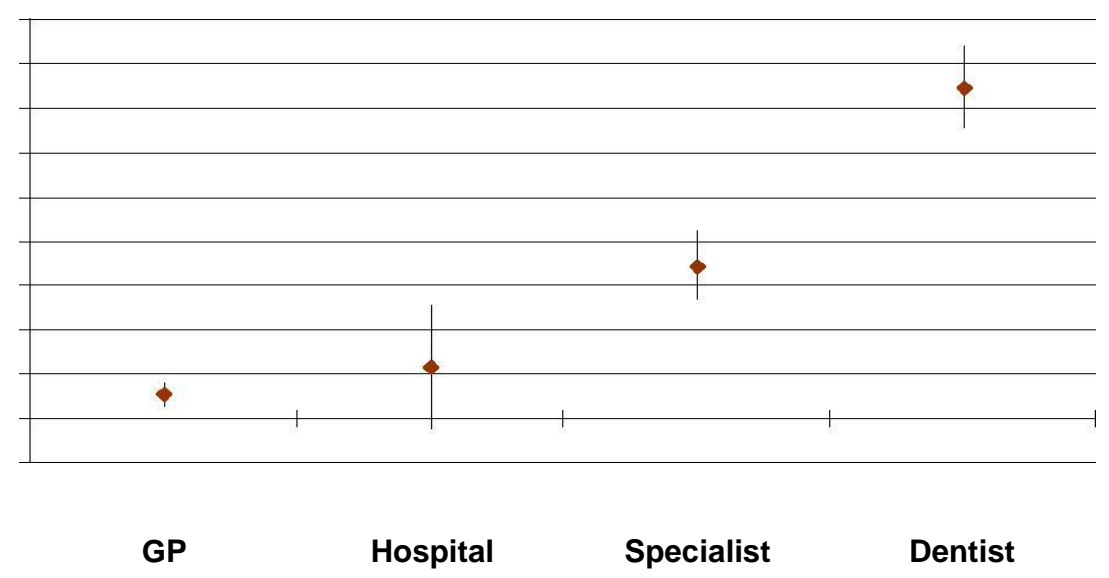

\section{Result}

The purpose of the present study was to investigate the degree of income-related inequity in the use of health services among older people Eastern Uttar Pradesh in the period 1997-2003 using a panel data approach. Results support the existence of significant pro-rich inequity to varying degrees in GP, outpatient and dental care. No significant income-related inequity could be found for inpatient admissions.

\section{References}

- Adamson, J, Ben-Shlomo, Y, Chaturvedi, N and Donovan, J. (2003) 'Ethnicity, socioeconomic position and gender-do they affect reported health-care seeking behaviour?'Social Science and Medicine 57: 895-904.

- Bago d'Uva, T. (2005) 'Latent class models for useof primary care: evidence from 11 waves of the BHPS'. Health Economics 14(9): 873-892.

- Barer, M, Manga, P and Shillington, R.(1982) Income class and hospital use in Ontario. Toronto, Ontario Economic Council

- Batchelor, PA. (2004) 'Changes in self-reported attendance of British adults for dental check-ups between 1991 and 2000'. Primary Dental Care 11(4): 125-130.

- Blaxter, M. (1985) 'Self-definition of health status and consulting rates in primary care'. Quarterly Journal of Social Affairs 1: 131-171.

- British Society of Gerodontology.(2005) Meeting the Challenges of Oral Health for Older People: A Strategic Review was commissioned and funded by the Department of Health. England, British Society of Gerodontology

- Dening, TR, Chi, LY, Brayne, C, Huppert, FA, Paykel, ES and O'Connor, DW. (1998) 'Changes in self-rated health, disability and contact with services in a very elderly cohort: a 6-year follow-up study'. Age and Ageing 27: 23-33.

- Dixon, A, Le Grand, J, Henderson, J, Murray, R and Poteliakhoff, E. (2006) 'Is the British National Health Service equitable? The evidence on socio-economic differences in utilisation'. Journal of Health Services Research and Policy Forthcoming 
- Evandrou, M.(2003) 'Growing old in London: socio-economic inequalities'. SAGE Discussion paper no. 15. London, Stimulating Social Policy in an Ageing Society (SAGE)

- Goddard, M and Smith, P. (2001) 'Equity of access to health care services: theory and evidence from UK'. Social Science and Medicine 53: 1149-1162. 